UCRHEP-T314

November 2001

\title{
Unified Supersymmetric Model of Naturally Small Dirac Neutrino Masses and the Axionic Solution of the Strong CP Problem
}

\author{
Ernest Ma \\ Physics Department, University of California, Riverside, California 92521
}

\begin{abstract}
Using the particle content of the fundamental 27 supermultiplet of $E_{6}$, naturally small Dirac neutrino masses are obtained in the context of $S U(3)_{C} \times S U(2)_{L} \times U(1)_{Y} \times$ $U(1)_{\chi}$, where $U(1)_{\chi}$ comes from the decomposition $E_{6} \rightarrow S O(10) \times U(1)_{\psi}$, then $S O(10) \rightarrow S U(5) \times U(1)_{\chi}$. New observable consequences are predicted at the $\mathrm{TeV}$ scale. An axionic solution of the strong $\mathrm{CP}$ problem may be included at no extra cost.
\end{abstract}


With the present experimental evidence [1, 2, [2] on neutrino oscillations, the notion that neutrinos should be massive is no longer in dispute. The next question is whether neutrino masses are Majorana or Dirac. Experimentally, the nonobservation of neutrinoless double beta decay at the $0.2 \mathrm{eV}$ level [4] is unable to settle this issue, but there are very strong and convincing theoretical reasons to believe that neutrino masses should be Majorana. On the other hand, if the theoretical context is changed, naturally small Dirac neutrino masses are possible, as shown below.

To obtain a Dirac mass, the left-handed neutrino $\nu_{L}$ must be paired with a right-handed singlet $N_{R}$. Two problems arise immediately. (i) There is no symmetry to prevent $N_{R}$ from acquiring a large Majorana mass. (ii) Even if such a symmetry (such as additive lepton number) is imposed, an extremely small Yukawa coupling (less than $10^{-11}$ ) is still needed to satisfy the experimental bound $m_{\nu}<\mathrm{a}$ few $\mathrm{eV}$. The usual resolution of these problems is to take advantage of $(i)$ to make $m_{N}$ very large, so that the famous canonical seesaw mechanism [5] makes $m_{\nu}=m_{D}^{2} / m_{N}$. Now (ii) is also not a problem because the Yukawa coupling for the Dirac mass $m_{D}$ is no longer required to be very small.

In this paper a new scenario is proposed where $(i) N_{R}$ is naturally prevented from having a Majorana mass and $(i i) m_{D}$ is small without having a small Yukawa coupling [6, 7]. This is possible because the theoretical framework used will be that of superstring-inspired $E_{6}$ [8]. As a bonus, the axionic solution [9] of the strong CP problem may also be included.

The starting point is the gauge group $E_{6}$ and its decomposition $E_{6} \rightarrow S O(10) \times U(1)_{\psi}$, then $S O(10) \rightarrow S U(5) \times U(1)_{\chi}$. It is often assumed that at $\mathrm{TeV}$ energies, a linear combination of $U(1)_{\psi}$ and $U(1)_{\chi}$ remains [10] in addition to the standard $S U(3)_{C} \times S U(2)_{L} \times U(1)_{Y}$. It is usually also assumed that three complete matter supermultiplets of the fundamental $\underline{27}$ representation of $E_{6}$ are present at these energies, which include the known three families of quarks and leptons as well as other new particles. Under the subgroup $S U(5) \times U(1)_{\psi} \times U(1)_{\chi}$, 
the particle content of each supermultiplet is given by

$$
\begin{aligned}
\underline{27} & =(10 ; 1,-1)\left[(u, d), u^{c}, e^{c}\right]+\left(5^{*} ; 1,3\right)\left[d^{c},\left(\nu_{e}, e\right)\right]+(1 ; 1,-5)\left[N^{c}\right] \\
& +(5 ;-2,2)\left[h,\left(E^{c}, N_{E}^{c}\right)\right]+\left(5^{*} ;-2,-2\right)\left[h^{c},\left(\nu_{E}, E\right)\right]+(1 ; 4,0)[S]
\end{aligned}
$$

where the $\mathrm{U}(1)$ charges refer to $2 \sqrt{6} Q_{\psi}$ and $2 \sqrt{10} Q_{\chi}$. Note that the known quarks and leptons are contained in $(10 ; 1,-1)$ and $\left(5^{*} ; 1,3\right)$, and the two Higgs scalar doublets are represented by $\left(\nu_{E}, E\right)$ and $\left(E^{c}, N_{E}^{c}\right)$. Since $N^{c}$ and $S$ are singlets under $S U(5)$, one linear combination will be trivial under the assumed low-energy gauge group, i.e. $S U(3)_{C} \times$ $S U(2)_{L} \times U(1)_{Y} \times U(1)_{\alpha}$ with $Q_{\alpha}=Q_{\psi} \cos \alpha+Q_{\chi} \sin \alpha$. For the choice $\tan \alpha=\sqrt{1 / 15}$, the $U(1)_{N}$ model [11, 12 is obtained, for which $N^{c}$ is trivial, thus allowing it to acquire a large Majorana mass. Combining this with the existing term $\left(\nu_{e} N_{E}^{c}-e E^{c}\right) N^{c}$, the usual seesaw Majorana neutrino mass may then be obtained.

Consider now the case $\sin \alpha=1$, i.e. the $U(1)_{\chi}$ model. This allows $S$ to have a large Majorana mass, but not $N^{c}$. Hence the only apparent way that $\nu_{e}$ may become massive is to pair up with $N^{c}$ to form a Dirac neutrino with mass proportional to the vacuum expectation value $(\mathrm{VEV})$ of the scalar component of $N_{E}^{c}$. If the latter is of the order of the electroweak symmetry breaking scale, i.e. $10^{2} \mathrm{GeV}$, then an extremely small Yukawa coupling is required. This is in fact the prevailing working ansatz of all $U(1)_{\alpha}$ models except $U(1)_{N}$. However, there is a very simple and natural solution. If $\tilde{N}_{E}^{c}$ has $m^{2}>0$ with $m$ large, then its VEV can be very small [6, 7]. This is precisely the case in the $U(1)_{\chi}$ model, where $\nu_{E} N_{E}^{c}-E E^{c}$ is an allowed term.

There are 11 generic terms 13 in the superpotential of such $E_{6}$ models. They are

$$
\begin{aligned}
& \text { (1) } \hat{Q} \hat{u}^{c} \hat{\bar{E}}=\left(\hat{u} \hat{N}_{E}^{c}-\hat{d} \hat{E}^{c}\right) \hat{u}^{c} \\
& \text { (2) } \hat{Q} \hat{d}^{c} \hat{E}=\left(\hat{u} \hat{E}-\hat{d} \hat{\nu}_{E}\right) \hat{d}^{c} \\
& \text { (3) } \quad \hat{L} \hat{e}^{c} \hat{E}=\left(\hat{\nu}_{e} \hat{E}-\hat{e} \hat{\nu}_{E}\right) \hat{e}^{c}
\end{aligned}
$$


(4) $\hat{S} \hat{E} \hat{\bar{E}}=\left(\hat{\nu}_{E} \hat{N}_{E}^{c}-\hat{E} \hat{E}^{c}\right) \hat{S}$,

(5) $\hat{S} \hat{h} \hat{h}^{c}$,

(6) $\hat{L} \hat{N}^{c} \hat{\bar{E}}=\left(\hat{\nu}_{e} \hat{N}_{E}^{c}-\hat{e} \hat{E}^{c}\right) \hat{N}^{c}$,

(7) $\hat{Q} \hat{L} \hat{h}^{c}=\left(\hat{u} \hat{e}-\hat{d}_{\hat{\nu}}\right) \hat{h}^{c}$,

(8) $\hat{u}^{c} \hat{e}^{c} \hat{h}$,

(9) $\hat{d}^{c} \hat{N}^{c} \hat{h}$,

(10) $\hat{Q} \hat{Q} \hat{h}=(\hat{u} \hat{d}-\hat{d} \hat{u}) \hat{h}$,

(11) $\hat{u}^{c} \hat{d}^{c} \hat{h}^{c}$.

To prevent rapid proton decay, some terms must be absent. This is usually accomplished by the imposition of an exactly conserved discrete symmetry, such as the well-known $R$ parity. Here the choice is

$$
Z_{3} \times U(1)_{P Q}
$$

Under $Z_{3}$ with $\omega^{3}=1, \hat{E}_{1,2}, \hat{\bar{E}}_{1,2}$ transform as $\omega ; \hat{u}^{c}, \hat{d}^{c}, \hat{e}^{c}$ as $\omega^{2}$; and all other superfields as 1. Under $U(1)_{P Q}$, the only superfields with nonzero charges are $\hat{h}, \hat{h}^{c}, \hat{S}_{1}, \hat{S}_{2}, \hat{S}_{3}$ with charges $1 / 2,1 / 2,-1,2,-2$ respectively. This means that the terms (7) to (11) are all forbidden, the term (6) involves only $\hat{\bar{E}}_{3}$, the term (5) involves only $\hat{S}_{1}$, and the term (4) is forbidden, but since $\hat{S}$ is trivial under $U(1)_{\chi}$, the soft term

$$
\hat{E} \hat{\bar{E}}=\hat{\nu}_{e} \hat{N}_{E}^{c}-\hat{E} \hat{E}^{c}
$$

by itself is allowed. Note that only one term, i.e. $\hat{E}_{3} \hat{\bar{E}}_{3}$, is invariant under $Z_{3}$. All other $\hat{E} \hat{\bar{E}}$ terms will break $Z_{3}$ but only softly.

The superpotential of this model is then given by

$$
\begin{aligned}
\hat{W} & =\mu_{i j} \hat{E}_{i} \hat{\bar{E}}_{j}+f_{(1,2) i j}^{(u)} \hat{Q}_{i} \hat{u}_{j}^{c} \hat{\bar{E}}_{1,2}+f_{(1,2) i j}^{(d)} \hat{Q}_{i} \hat{d}_{j}^{c} \hat{E}_{1,2}+f_{(1,2) i j}^{(e)} \hat{L}_{i} \hat{e}_{j}^{c} \hat{E}_{1,2} \\
& +f_{i j}^{(N)} \hat{L}_{i} \hat{N}_{j}^{c} \hat{\bar{E}}_{3}+f_{i j}^{(h)} \hat{S}_{1} \hat{h}_{i} \hat{h}_{j}^{c}+m_{2} \hat{S}_{2} \hat{S}_{3}+f_{2} \hat{S}_{2} \hat{S}_{1} \hat{S}_{1}
\end{aligned}
$$


The anomalous global $U(1)_{P Q}$ is spontaneously broken at the intermediate scale $m_{2}$ so that an "invisible" axion will emerge to solve the strong CP problem. The $U(1)_{P Q}$ charges of $\hat{S}_{1,2,3}$ are chosen so that $S_{1}$ may acquire a large $\operatorname{VEV}\left(\sim m_{2} \sim 10^{9}\right.$ to $\left.10^{12} \mathrm{GeV}\right)$ without breaking the supersymmetry of the entire theory at that scale. Details are contained in Ref.[14]. Because the usual quarks and leptons here do not transform under $U(1)_{P Q}$, the axion of this model is of the KSVZ type [15], whereas that of Ref.[14] is of the DFSZ type [16].

Note that $U(1)_{P Q}$ here serves the dual purpose of solving the problem of rapid proton decay as well. Note also that the choice of $U(1)_{\chi}$ as the extra gauge symmetry is the only one which allows that to work. It also serves the purpose of allowing the term $\hat{E} \hat{\bar{E}}$ and the choice of $Z_{3}$ allows only $\hat{\bar{E}}_{3}$ to couple to $\hat{N}^{c}$, with a large mass for $\hat{E}_{3} \hat{\bar{E}}_{3}$. The Dirac mass linking $\nu_{e}$ to $N^{c}$ is proportional to the VEV of the scalar component of $\hat{\bar{E}}_{3}$, which may then be very small [6, 7], as shown below.

Consider the following Higgs potential of 4 scalar doublets $H_{1,2,3,4}$ representing the scalar components of $\hat{E}_{1}, \hat{\bar{E}}_{1}, \hat{E}_{3}, \hat{\bar{E}}_{3}$ respectively 17] (assuming that $\hat{E}_{2}$ and $\hat{\bar{E}}_{2}$ have no VEV):

$$
\begin{aligned}
V= & \sum_{i} m_{i}^{2} H_{i}^{\dagger} H_{i}+\left[m_{13}^{2} H_{1}^{\dagger} H_{3}+m_{24}^{2} H_{2}^{\dagger} H_{4}\right. \\
& \left.+m_{12}^{2} H_{1} H_{2}+m_{14}^{2} H_{1} H_{4}+m_{32}^{2} H_{3} H_{2}+m_{34}^{2} H_{3} H_{4}+\text { h.c. }\right] \\
+ & \frac{1}{2}\left(\frac{g_{1}^{2}}{4}+\frac{g_{\chi}^{2}}{10}\right)\left[-H_{1}^{\dagger} H_{1}+H_{2}^{\dagger} H_{2}-H_{3}^{\dagger} H_{3}+H_{4}^{\dagger} H_{4}\right]^{2} \\
+ & \frac{1}{2} g_{2}^{2} \sum_{\alpha}\left|\sum_{i} H_{i}^{\dagger} \tau_{\alpha} H_{i}\right|^{2},
\end{aligned}
$$

where $\tau_{\alpha}(\alpha=1,2,3)$ are the usual $\mathrm{SU}(2)$ representation matrices. Let the VEV's of $H_{i}$ be $v_{i}$, then the minimum of $V$ is

$$
\begin{aligned}
V_{\text {min }} & =\sum_{i} m_{i}^{2} v_{i}^{2}+2 m_{12}^{2} v_{1} v_{2}+2 m_{13}^{2} v_{1} v_{3}+2 m_{14}^{2} v_{1} v_{4}+2 m_{24}^{2} v_{2} v_{4}+2 m_{32}^{2} v_{2} v_{3}+2 m_{34}^{2} v_{3} v_{4} \\
& +\frac{1}{8}\left(g_{1}^{2}+g_{2}^{2}+\frac{2 g_{\chi}^{2}}{5}\right)\left(v_{1}^{2}-v_{2}^{2}+v_{3}^{2}-v_{4}^{2}\right)^{2}
\end{aligned}
$$


where all parameters have been assumed real for simplicity. The 4 equations of constraint are

$$
\begin{aligned}
0 & =m_{1}^{2} v_{1}+m_{12}^{2} v_{2}+m_{13}^{2} v_{3}+m_{14}^{2} v_{4}+\frac{1}{4}\left(g_{1}^{2}+g_{2}^{2}+\frac{2 g_{\chi}^{2}}{5}\right) v_{1}\left(v_{1}^{2}-v_{2}^{2}+v_{3}^{2}-v_{4}^{2}\right), \\
0 & =m_{2}^{2} v_{2}+m_{12}^{2} v_{1}+m_{24}^{2} v_{4}+m_{32}^{2} v_{3}-\frac{1}{4}\left(g_{1}^{2}+g_{2}^{2}+\frac{2 g_{\chi}^{2}}{5}\right) v_{2}\left(v_{1}^{2}-v_{2}^{2}+v_{3}^{2}-v_{4}^{2}\right), \\
0 & =m_{3}^{2} v_{3}+m_{13}^{2} v_{1}+m_{32}^{2} v_{2}+m_{34}^{2} v_{4}+\frac{1}{4}\left(g_{1}^{2}+g_{2}^{2}+\frac{2 g_{\chi}^{2}}{5}\right) v_{3}\left(v_{1}^{2}-v_{2}^{2}+v_{3}^{2}-v_{4}^{2}\right), \\
0 & =m_{4}^{2} v_{4}+m_{24}^{2} v_{2}+m_{14}^{2} v_{1}+m_{34}^{2} v_{3}-\frac{1}{4}\left(g_{1}^{2}+g_{2}^{2}+\frac{2 g_{\chi}^{2}}{5}\right) v_{4}\left(v_{1}^{2}-v_{2}^{2}+v_{3}^{2}-v_{4}^{2}\right) .
\end{aligned}
$$

Since $m_{3}^{2} \sim m_{4}^{2} \sim \mu_{33}^{2}, m_{13}^{2} \sim \mu_{13} \mu_{33}$, and $m_{24}^{2} \sim \mu_{31} \mu_{33}$ are the only parameters which have contributions involving the large mass $\mu_{33}$, it is clear that Eqs. (20) and (21) have the solution

$$
v_{3} \simeq-\frac{m_{13}^{2} v_{1}}{m_{3}^{2}}, \quad v_{4} \simeq-\frac{m_{24}^{2} v_{2}}{m_{4}^{2}} .
$$

They may then be of order $0.1 \mathrm{eV}$ if $m_{3,4} \sim \mu_{33} \sim 10^{15} \mathrm{GeV}$ (i.e. close to a possible grandunification mass scale), and $\mu_{13}, \mu_{31} \sim M_{S U S Y} \sim 1 \mathrm{TeV}$. Setting $v_{3}=v_{4}=0$ in Eqs. (18) and (19), the usual conditions of the minimal supersymmetric standard model are obtained except for the additional terms due to $g_{\chi}$.

Now $U(1)_{\chi}$ also undergoes spontaneous symmetry breaking through the VEV of one linear combination of the $3\left(\tilde{N}^{c}\right)$ 's. As a result, there appear a new massive neutral gauge boson $Z^{\prime}$, the corresponding scalar boson $\sqrt{2} R e \tilde{N}^{c}$, and the Dirac fermion which comes from the pairing of $\tilde{z}^{\prime}$ and $N^{c}$, all having the mass $(\sqrt{5} / 2) g_{\chi}\left\langle\tilde{N}^{c}\right\rangle$ 18. Hence only $2\left(N^{c}\right)$ 's remain and they combine with 2 of the $3 \nu$ 's to form 2 light Dirac neutrinos. The remaining $\nu$ gets a negligible Majorana mass from the allowed supersymmetry-breaking soft Majorana mass of $\tilde{z}^{\prime}$. A satisfactory framework is thus established for describing the oscillations of 2 light Dirac neutrinos and 1 essentially massless Majorana neutrino.

At the TeV energy scale, this model is verifiable experimentally by its many unique 
predictions. First, there must be a $Z^{\prime}$ gauge boson with couplings to quarks and leptons according to Eq. (1). In particular, it will have invisible decays to neutrinos given by

$$
\frac{\Gamma\left(Z^{\prime} \rightarrow \bar{\nu} \nu+\bar{N}^{c} N^{c}\right)}{\Gamma\left(Z^{\prime} \rightarrow l^{+} l^{-}\right)}=\frac{77}{30}
$$

There are likely to be 4 Higgs doublets, instead of 2 , and definitely not 6 . There should not be exotic quarks (i.e. $h$ and $h^{c}$ ) because they are predicted to be very heavy with masses at the axion scale. The axion itself is of course very light and very difficult to detect [19]. Its partners, the saxion and the axino, are likely to be at or below the $\mathrm{TeV}$ scale and may also be components of the dark matter of the Universe. Lepton number is violated through $\left\langle\tilde{N}^{c}\right\rangle$, but since $\hat{N}^{c}$ only appears in Eq. (15) with the very heavy $\hat{\bar{E}}_{3}$, this violation is highly suppressed. Thus my proposed model evades the general conclusion of Ref. [12] regarding $E_{6}$ subgroups that only $U(1)_{N}$ [11] and the skew left-right model [20] do not have lepton-number violating interactions at the $\mathrm{TeV}$ scale which would erase any preexisting lepton or baryon asymmetry of the Universe.

In conclusion, a new unified supersymmetric model has been proposed which has the following desirable properties.

(1) Its particle content comes from 3 complete fundamental $\underline{27}$ representations of $E_{6}$, which may be the remnant of an underlying superstring theory.

(2) Its low-energy gauge group is $S U(3)_{C} \times S U(2)_{L} \times U(1)_{Y} \times U(1)_{\chi}$, where $U(1)_{\chi}$ comes from $E_{6} \rightarrow S O(10) \rightarrow S U(5) \times U(1)_{\chi}$.

(3) It has the additional symmetry $Z_{3} \times U(1)_{P Q}$ which serves many purposes, including that of preventing rapid proton decay. $Z_{3}$ is softly broken; $U(1)_{P Q}$ is spontaneously broken.

(4) Naturally small Dirac neutrino masses [21, 22] come from the $\hat{L} \hat{N}^{c} \hat{\bar{E}}_{3}$ term of Eq. (15) because $\tilde{\bar{E}}_{3}$ has a very small VEV, using the mechanism [6, 4] of a large positive $m^{2}$ close to a possible grand-unification mass scale for $\tilde{\bar{E}}_{3}$, as shown by Eq. (22). 
(5) The 3 singlet superfields $\hat{S}_{1,2,3}$, which do not transform under $U(1)_{\chi}$, are chosen [14] to obtain an axionic solution of the strong CP problem, such that $f_{a}>>M_{S U S Y}$.

(6) This model predicts a definite supersymmetric particle structure associated with the

extra $U(1)_{\chi}$ gauge symmetry at the $\mathrm{TeV}$ scale, which should be accessible in near-future high-energy accelerators.

(7) It is the only model to date which incorporates naturally small Dirac neutrino masses with the axionic solution of the strong CP problem in a comprehensive theoretical framework of all particle interactions.

This work was supported in part by the U. S. Department of Energy under Grant No. DEFG03-94ER40837.

\section{References}

[1] S. Fukuda et al., Super-Kamiokande Collaboration, Phys. Rev. Lett. 85, 3999 (2000) and references therein.

[2] S. Fukuda et al., Super-Kamiokande Collaboration, Phys. Rev. Lett. 86, 5656 (2001) and references therein. See also Q. R. Ahmad et al., SNO Collaboration, Phys. Rev. Lett. 87, 071301 (2001).

[3] A. Aguilar et al., hep-ex/0104049; see also G. B. Mills (for the LSND Collaboration), Nucl. Phys. Proc. Suppl. 91, 198 (2001) and references therein.

[4] L. Baudis et al., Phys. Rev. Lett. 83, 41 (1999).

[5] M. Gell-Mann, P. Ramond, and R. Slansky, in Supergravity, edited by P. van Nieuwenhuizen and D. Z. Freedman (North-Holland, Amsterdam, 1979), p. 315; T. Yanagida, in Proceedings of the Workshop on the Unified Theory and the Baryon Number in the 
Universe, edited by O. Sawada and A. Sugamoto (KEK, Tsukuba, Japan, 1979), p. 95;

R. N. Mohapatra and G. Senjanovic, Phys. Rev. Lett. 44, 912 (1980).

[6] E. Ma, Phys. Rev. Lett. 86, 2502 (2001).

[7] E. Ma, Phys. Lett. B516, 165 (2001).

[8] For a review, see for example J. L. Hewett and T. G. Rizzo, Phys. Rept. 183, 193 (1989).

[9] R. D. Peccei and H. R. Quinn, Phys. Rev. Lett. 38, 1440 (1977); S. Weinberg, ibid., 40, 223 (1978); F. Wilczek, ibid., 40, 279 (1978).

[10] See for example G. C. Cho, K. Hagiwara, and Y. Umeda, Nucl. Phys. B531, 65 (1998); erratum: B555, 651 (1999).

[11] E. Ma, Phys. Lett. B380, 286 (1996).

[12] T. Hambye, E. Ma, M. Raidal, and U. Sarkar, Phys. Lett. B512, 373 (2001).

[13] E. Ma, Phys. Rev. Lett. 60, 1363 (1988).

[14] E. Ma, Phys. Lett. B514, 330 (2001).

[15] J. E. Kim, Phys. Rev. Lett. 43, 103 (1979); M. A. Shifman, A. I. Vainshtein, and V. I. Zakharov, Nucl. Phys. B166, 493 (1980).

[16] M. Dine, W. Fischler, and M. Srednicki, Phys. Lett. B104, 199 (1981); A. R. Zhitnitsky, Sov. J. Nucl. Phys. 31, 260 (1980).

[17] E. Ma, Phys. Rev. D64, 097302 (2001).

[18] E. Keith and E. Ma, Phys. Rev. D54, 3587 (1996). 
[19] L. J. Rosenberg and K. A. van Bibber, Phys. Rept. 325, 1 (2000).

[20] E. Ma, Phys. Rev. D36, 274 (1987); K. S. Babu, X.-G. He, and E. Ma, Phys. Rev. D36, 878 (1987).

[21] For other recent discussions, see for example P. P. Divakaran and G. Rajasekaran, Mod. Phys. Lett. A14, 913 (1999); P. Q. Hung, Phys. Rev. D59, 113008 (1999); D62, 053015 (2000).

[22] Another approach is to use extra dimensions: N. Arkani-Hamed, S. Dimopoulos, G. Dvali, and J. March-Russell, hep-ph/9811448; K. R. Dienes, E. Dudas, and T. Gherghetta, Nucl. Phys. B557, 25 (1999); G. Barenboim, G. C. Branco, A. de Gouvea, and M. N. Rebelo, Phys. Rev. D64, 073005 (2001). 